\title{
Kombine Derin Öğrenme Tabanlı Epileptik Nöbet Teşhisi
}

\author{
Muhammet Varl ${ }^{1}$, Hakan Y1lmaz ${ }^{2 *}$ \\ ${ }^{1}$ Karabük Üniversitesi, Lisansüstü Eğitim Enstitüsü Biyomedikal Mühendisliği ABD, Karabük, Türkiye, (ORCID: 0000-0003-3902-4504), \\ muhammetvar1329@gmail.com \\ $2^{2^{*}}$ Karabük Üniversitesi, Mühendislik Fakültesi, Tıp Mühendisliği, Karabük, Türkiye (ORCID: 0000-0002-8553-388X), hakanyilmaz@karabuk.edu.tr
}

(1st International Conference on Applied Engineering and Natural Sciences ICAENS 2021, November 1-3, 2021)

(DOI: 10.31590/ejosat.1013489)

ATIF/REFERENCE: Varlı, M., Yılmaz, H. (2021). Kombine Derin Öğrenme Tabanlı Epileptik Nöbet Teşhisi. Avrupa Bilim ve Teknoloji Dergisi, (28), 1210-1216.

\section{$\ddot{O} \mathbf{z}$}

Epilepsi hastalı̆̆ı yaygın nörolojik hastalıklardan bir tanesi olarak öne çıkmaktadır. Epilepsi hastalığının teşhisinde elektroensefalografi (EEG) kullanılarak beynin sinirsel aktivitesi gözlemlenir ve bu da epilepsi hastalığının teşhisine olanak sağlar. Günümüzde genel olarak biyolojik sinyallerden hastalık teşhisinde klasik makine öğrenmesi yöntemleri sıklıkla kullanılmakla birlikte son yıllarda derin öğrenme yapıları ön plana çıkmaktadır. Derin öğrenme ağları sinyallerden özellik çıkarımına gerek duymaması, özellikler için ek bir çaba gerektirmemesi, insan kaynaklı hesaplama hatalarının önüne geçmesi ve zaman kaybının önüne geçmesi açısından klasik makine öğrenmesi yöntemlerine göre daha avantajlı bir konuma gelmektedir. Bu çalı̧şada, zaman serisi EEG sinyalini, zaman-frekans bileşenlerini temsil edecek görüntüleri ve ham EEG sinyallerinin sayısal değerlerini kullanarak epilepsi nöbet aktivitesini otomatik bir şekilde tespit eden kombine bir derin öğrenme modeli üzerine çalışılmıştır. Çalışmada Bonn Üniversitesinin halka açık epilepsi veri seti kullanılmıştır. Veri seti sağlıklı ve epilepsi hastası insanlardan kaydedilen A,B,C,D,E şeklinde etiketlenmiş EEG kayıtlarını içermektedir. Bu çalışmada EGG sinyallerinin zaman dizisini ve zamana bağlı EEG sinyallerinin zaman-frekans-görüntü dönüşümlerini kullanarak kombine bir model ortaya koyulmuştur. Sinyalleri görüntülere dönüştürmede CWT ve STFT yöntemleri kullanılmıştır. Oluşturulan modelin CNN girdilerinde STFT görüntüleri kullanıldığında ikili sınıflandırma için \%99.47 doğruluk oranı elde edilmiştir. CWT görüntüleri ile ise \%99.27 doğruluk oranına ulaşılmıştır. Elde edilen model, EEG verilerinde epilepsi nöbet aktivitesinin olup olmadığını yüksek başarı ile tespit edebilmektedir.

Anahtar Kelimeler: Epilepsi, Evrişimli Sinir Ağı, Yinelenen Sinir Ağı, EEG, Epileptik nöbet teşhisi

\section{Combined Deep Learning Based Epileptic Seizure Diagnosis}

\begin{abstract}
Epilepsy stands out as one of the common neurological diseases. In the diagnosis of epilepsy, the neural activity of the brain is observed using electroencephalography (EEG), which allows the diagnosis of epilepsy disease. Although classical machine learning methods are frequently used in the diagnosis of diseases from biological signals, deep learning structures have come to the fore in recent years. Deep learning networks are in a more advantageous position than classical machine learning methods in terms of not requiring feature extraction from signals, requiring no additional effort for features, preventing human-induced computational errors, and preventing time loss. In this study, a combined deep learning model that automatically detects epileptic seizure activity using images representing the time-frequency components of the time domain EEG signal and numerical values of the raw EEG signals was studied. The public epilepsy dataset of the University of Bonn was used in the study. The dataset includes EEG recordings labelled as A, B, C, D, E recorded from healthy and epileptic people. In this study, we made a combined model using the time sequence of EGG signals and timefrequency-image transformations of time-dependent EEG signals. We used CWT and STFT to convert signals to images. We achieved 99.47\% accuracy for binary classification when we used STFT images in the CNN inputs of our model. With CWT images, we performed an accuracy rate of $99.27 \%$. The model obtained can detect with high success whether there is epileptic seizure activity in EEG data.
\end{abstract}

Keywords: Epilepsy, Convolutional Neural Network, Recurrent Neural Network, EEG, Epileptic Seizure Diagnos

\footnotetext{
* Sorumlu Yazar: hakanyilmaz@karabuk.edu.tr
} 


\section{Giriş}

Epilepsi hastalığı yaklaşık olarak dünya genelinde 70 milyondan çok kişiyi etkilemektedir (Singh \& Trevick, 2016). Bu kadar çok sayıda insanı etkisi altına almasından dolayı epilepsi, dünya çapında oldukça fazla insanın muzdarip olduğu bir nörolojik hastalık olarak bilinmektedir. Epilepsi beynin olağan elektriksel aktivitesi dışında sinir hücrelerinde meydana gelen anormal elektriksel boşalımlar sonucu ortaya çıkabilen kronik bir rahatsızlıktır (Beghi, 2020). Epilepsi nöbetleri aniden meydana gelebilir ve hastaların günlük yaşantılarını olumsuz yönde etkiler. EEG beynin elektriksel aktivitesinin gözlemlenmesinde kullanılan invaziv olmayan klinik bir tanı yöntemi olarak kabul edilir. EEG sinyalleri düşük uzamsal çözünürlüğe sahip olmasının aksine yüksek zamansal çözünürlüğe sahiptir ve bu da sinyal analizi için önemli bir avantaj sağlamaktadır (van Mierlo, Vorderwülbecke, Staljanssens, Seeck, \& Vulliémoz, 2020). EEG sinyallerinin tipik olarak 0-63 Hz. arasında bilgi taşıdığ 1 düşünülebilir. EEG sinyallerinin frekans bantları genellikle Delta $(0,1-4 \mathrm{~Hz}$ veya $0,5-4 \mathrm{~Hz})$, Teta $(4-8 \mathrm{~Hz})$, Alfa $(8-14 \mathrm{~Hz})$, Beta (14-30 Hz), Gama (30-63 Hz) olarak kabul görür (Bajaj, 2020). EEG verileri ile epilepsi, otizm, parkinson gibi birçok nörolojik rahatsızlığın tespiti mümkündür. Nörolojik rahatsızlıkların otomatik teşhisi ile ilgili birçok çalışma mevcuttur (Brian et al., 2021; Shi, Wang, Wang, Liu, \& Yan, 2019; Xu et al., 2020). Epilepsi tespitinde kullanılmak üzere birçok açık kaynak veri seti mevcuttur (Siddiqui, Morales-Menendez, Huang, \& Hussain, 2020). Otomatik epilepsi tespitinde genel olarak klasik makine öğrenmesi yaklaşımı ve derin öğrenme yaklaşımı olmak üzere iki tip yaklaşım mevcuttur. Birinci yaklaşımda EEG sinyallerinden çeşitli istatistiksel hesaplamalar yapılarak özellikler çıkarılır. Bu istatistiksel parametrelere genel olarak katsayıların mutlak değerlerinin ortalaması, katsayıların mutlak değerlerinin maksimumu ve minimumu, katsayıların kuvvetlerinin ortalaması, standart sapma, varyans, çarpıklık gibi parametreler örnek verilebilir (Hussain, 2018). Birinci yöntem ile yapılan daha önceki çalışmalardan Rao ve arkadaşları (2019) Random Forest (RF) sınıflandırıcı modeli (Ravi Kumar \& Srinivasa Rao, 2019) $94.1 \%$ doğruluk oranında başarı sağlamıştır. Shoka ve arkadaşları (2019) Support Vector Machine (SVM) tabanlı bir sistem kullanmıştır ve $98 \%$ doğruluk oranına ulaşmıştır (Shoka, Dessouky, El-Sherbeny, \& El-Sayed, 2019). Maryati ve arkadaşları (2019) yine SVM sınıflandırıcı kullanarak oluşturduğu modelde $91.4 \%$ doğruluk oranı elde etmiştir (Dwi Saputro et al., 2019). İkinci yaklaşımda EEG sinyallerinden manuel özellik çıkarımı yapılmaksızın bir derin öğrenme modeli ile otomatik bir şekilde özellik çıkarımı sağlanmaktadır. Derin öğrenme yaklaşımı klasik makine öğrenmesi yaklaşımına göre daha hızlı, hesaplama kaynaklı hatalardan arınmış bir sistem ortaya koymaktadır. Doğruluk değerleri ise son yıllardaki geliştirilen algoritma ve modeller ile kayda değer bir oranda yükselmiştir. Şekil 1'de iki yaklaşım özetlenmiştir.

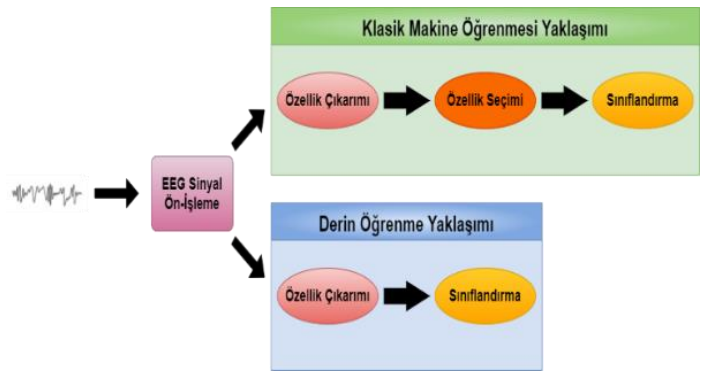

Şekil 1 Epilepsi tespitinde sınıflandırma yaklaşımları.
Bu çalışmada derin öğrenme modeli ile oluşturulan model üzerine yoğunlaşılmıştır. Çalışma içerisinde iki tane ayrı model oluşturulmuş ve bu modeller çıktı katmanından önce kombine edilerek birleştirilmiştir. Birinci model 2D Convolutional Neural Network (2D-CNN), diğeri ise Recurrent Neural Network (RNN) olarak ele alınmıştır. 2D-CNN için girdi verileri sinyallerin görüntüleri olarak belirlenmekle birlikte RNN için girdi verileri sinyalin ham yani işlenmemiş hali olarak belirlenmiştir. 2D-CNN yapısının girdisi olan verileri elde etmek için sinyallere çeşitli dönüşümler uygulanmıştır. Sinyallere Continuous wavelet transformation (CWT) uygulanması ile scalogram görüntüleri, Short-term Fourier transform (STFT) uygulanması ile spectrogram görüntüleri elde edilmiştir. Yapılan tüm çalışmada hem scalogram hem de spectrogram görüntüleri ayrı ayrı kullanılmıştır. RNN girdisi olarak ise sinyal değerleri normalize edilerek kullanılmıştır.

\section{Materyal ve Metot}

\subsection{Veri Seti}

Veri seti olarak Bonn Üniversitesi'nin sunmuş olduğu EEG kayıtlarını kullanılmıştır (Andrzejak et al., 2001). Veri seti A,B,C,D,E olmak üzere 5 set içermektedir. Her bir dizi toplam 23.6 saniye olmak üzere tek kanallı 100 EEG sinyal kaydından oluşmaktadır. EEG kayıtları her türlü kas hareketinden kaynaklı gürültülerden arındırılmıştır. A seti 5 sağlıklı kişiden gözleri açık bir şekilde elde edilmiştir. B seti yine aynı 5 sağlıklı kişiden gözleri kapalı bir şekilde elde edilmiştir. C,D,E setleri ise 5 epilepsi hastasından elde edilen kayıtları içermektedir. C seti epilepsi hastalarına ait nöbet öncesi olup zıt hemisferik bölgenin hipokampal yarım küresinden kayıtlar içermektedir. D seti epilepsi hastası olup nöbet öncesi epileptik bölgeden kayıtlar içermektedir. E seti ise epilepsi hastalarının epilepsi nöbeti geçirirken elde edilen kayıtları içermektedir. EEG verilerinin örnekleme frekans1 173.61 Hz.'dir. Veri seti için özet bilgiler Tablo 1'de verilmiştir.

Tablo 1. Veri seti bilgileri.

\begin{tabular}{|c|c|c|c|c|}
\hline $\mathbf{A}$ & B & $\mathbf{C}$ & D & $\mathbf{E}$ \\
\hline Sağlıklı & Sağlıklı & $\begin{array}{c}\text { Epilepsi } \\
\text { Hastasi }\end{array}$ & $\begin{array}{l}\text { Epilepsi } \\
\text { Hastasi }\end{array}$ & $\begin{array}{c}\text { Epilepsi } \\
\text { Hastas1 }\end{array}$ \\
\hline $\begin{array}{c}100 \\
\text { Tane }\end{array}$ & $\begin{array}{c}100 \\
\text { Tane }\end{array}$ & 100 Tane & 100 Tane & $\begin{array}{c}100 \\
\text { Tane }\end{array}$ \\
\hline $23.6 \mathrm{sn}$. & $23.6 \mathrm{sn}$. & $23.6 \mathrm{sn}$. & $23.6 \mathrm{sn}$. & $23.6 \mathrm{sn}$. \\
\hline $\begin{array}{c}\text { Gözler } \\
\text { Açık }\end{array}$ & $\begin{array}{l}\text { Gözler } \\
\text { Kapalı }\end{array}$ & $\begin{array}{c}\text { Nöbet } \\
\text { Öncesi } \\
\text { Hipokampal } \\
\text { Yarım } \\
\text { Küreden }\end{array}$ & $\begin{array}{c}\text { Nöbet } \\
\text { Öncesi } \\
\text { Epilepsi } \\
\text { Bölgesi } \\
\text { Üzerinden }\end{array}$ & $\begin{array}{l}\text { Nöbet } \\
\text { Anında }\end{array}$ \\
\hline
\end{tabular}

\subsection{Veri Ön İşleme}

Veri setinde her bir EEG kaydı 4097 veri noktasından oluşmaktadır. Şekil 2'de her bir setten birer EEG kaydının örnekleri gösterilmiştir. Veri sayısını çoğaltmak için veriler eşit parçalara bölünmüştür. 16 eşit parçaya bölmek için son veri noktaları silinerek 4096 veri noktası üzerinden işlem yapılmıştır ve toplamda her bir set için 1600 gözlem oluşturulmuştur. Böylece 8000 tane gözlem elde edilmiştir. Oluşturulan modelde RNN kısmı için kullanılacak ham sinyaller için normalizasyon işlemi uygulanmıştır. Bu işlemin asıl amacı veri setindeki olası 
aykırı değerlerden kaynaklı olan olumsuz etkileri önleyebilmektir. EEG sinyalleriyle zaman alanından zamanfrekans alanına geçiş yaparak 2D-CNN yapısı için gerekli olan girdi görüntüleri elde edilmiştir. Dönüşümler için Continuous Wavelet Transformation (CWT) ve Short-Term Fourier Transform (STFT) olmak üzere iki farklı yöntem kullanılmıştır.

\subsection{Continuous Wavelet Transformation (CWT)}

Wavelet Transform STFT'nin pencere boyutu seçiminden kaynaklanan çözünürlük kaybını azaltmayı amaçlamaktadır (Rajoub, 2020). CWT'de ana dalgacık denilen bir pencereleme fonksiyonu kullanılır. Bu pencereleme fonksiyonunun STFT'de kullanılan pencereleme fonksiyonundan farklılığı ölçeklenebilir olmasidir.

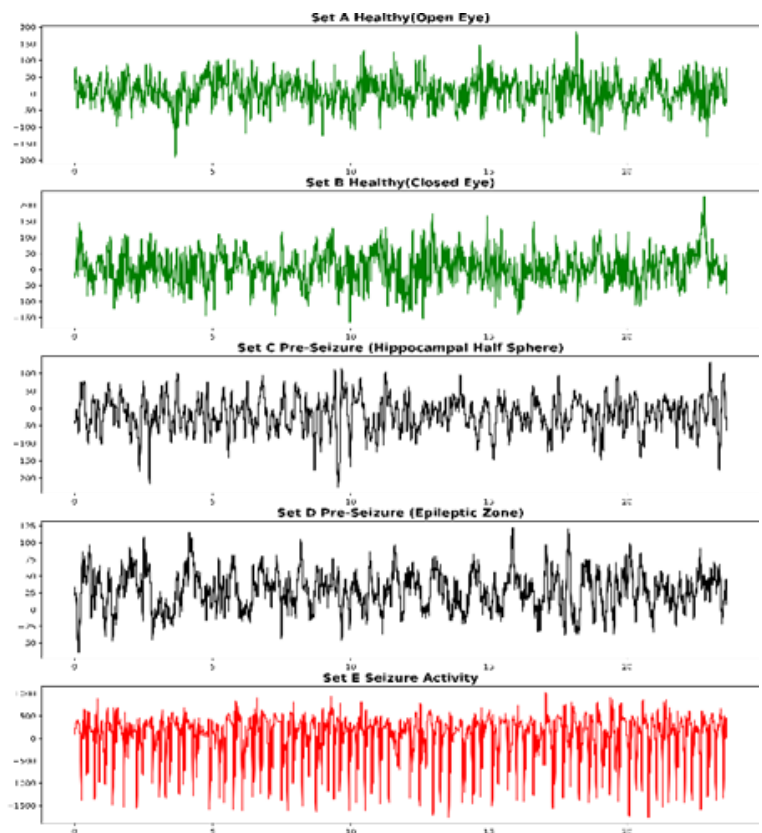

Şekil 2 A,B,C,D, E setlerinden EEG örnekleri.

CWT yapılırken dalgacık fonksiyonu zaman içerisinde kaydırılır ve Denklem (1)'de gösterildiği gibi ölçeklendirilir.

$$
\psi_{a, b}(t)=\frac{1}{\sqrt{a}} \psi\left(\frac{t-b}{a}\right) a, b \in R, \quad a \neq 0
$$

Burada a parametresi ölçeklendirme, b parametresi ise ötelemeyi yani kaydırmayı ifade eder. Düşük ölçek parametreleri sinyalleri sıkıştırırken yüksek ölçek parametreleri sinyalleri genişletir. Yüksek ölçek parametreleri düşük frekansları yakalarken, düşük ölçek parametreleri yüksek frekansları yakalar. CWT analiz edilecek olan sinyalin, dalgacik fonksiyonunun karmaşık eşleniği ile integrali şeklinde Denklem (2)'de gösterildiği gibi tanımlanır. Burada $\mathrm{f}(\mathrm{t})$ zamana bağlı sinyali ifade etmektedir.

$$
C W T\{f(t), a, b\}=\int_{-\infty}^{+\infty} \mathrm{f}(\mathrm{t}) \psi_{a, b}^{*}(t) d t
$$

EEG sinyalleri için düşük frekanstaki bilgilerde önemlidir. Çünkü EEG sinyallerinin Delta bandı oldukça düşük frekanslar içerir. $\mathrm{Bu}$ çalışmada ana dalgacık fonksiyonu olarak düşük frekanslarda etkili olan Morlet dalgacık fonksiyonu kullanılmıştır (Bajaj, 2020). CWT dönüşümü yaptıktan sonra katsayılar ve ölçek parametrelerine göre sinyalin scalogram diye adlandırılan görüntüsü oluşturulur. A,B,C,D,E setlerinin her birisi için 1600 'er tane görüntü oluşturulmuştur. Toplamda 8000 adet scalogram görüntüsü elde edilmiştir. Oluşan scalogram görüntülerinin her biri $77 \times 75$ boyutlarındadır.

\subsection{Short-Term Fourier transform (STFT)}

Fourier Transform (FT) zamana bağlı sinyalleri frekans alanına dönüştürmek için kullanılmaktadır. Yani sinyallerin spektral analizlerine olanak sağlar. FT sinyali sonsuz sayıda sinüs/kosinüs dalgasına ayrıştırabilir. Fakat FT'de zaman bilgisi kaybolur sadece sinyalin genlik ve frekansı elde edilir. Yani olayın tam olarak nerede olduğu bilinemez. FT sinyalin aniden patlamaları ve süreksizliği ile mücadele edemez. FT'nin bu dezavantajlarının önüne geçebilmek adına STFT geliştirilmiştir. FT durağan olmayan sinyalleri analiz etmek için uygun bir yöntem değildir (Kıymık, Güler, Dizibüyük, \& Akın, 2005). STFT, FT'de yaşanan problemlerin önüne geçebilmek adına belirli bir zamanda sinyalin sadece küçük bir bölümünü analiz ederek hareket eder. Durağan olmayan sinyaller küçük parçalara ayrılır ve bu parçaların art arda geldiği düşünülür. Böylece her bir parçaya FT uygulanır. Bu parçalar bir pencereleme fonksiyonu uygulayarak elde edilir. Bu yönteme sinyal pencereleme denir. STFT ile zamana bağlı sinyaller frekans ve zaman ekseninde ifade edilebilir. STFT'de pencere fonksiyonu seçiminde çözünürlük ikilemi ortaya çıkar. Pencere fonksiyonu seçilirken ya zaman ya da frekans çözünürlüğünden vazgeçilir. STFT'de pencereleme fonksiyonu olarak Hamming kullanılmıştır. STFT sonrasında spectrogram görüntüleri elde edilir. Spectrogram bir sinyalin zamanla değişen spektrumunun görsel temsilidir. A,B,C,D,E setlerinin her birisi için 1600 'er tane görüntü oluşturulmuştur. Toplamda 8000 adet spectrogram görüntüsü elde edilmiştir. Oluşan spectrogram görüntülerinin her biri 37x38 boyutlarındadır. Sinyal görüntülerinin elde edildiği CWT ve STFT yöntemlerinin 4096 veri noktasına sahip her bir EEG kaydı için nasıl işleme alındığı ile ilgili olarak Şekil 3'te özet bir diyagram görselleştirilmiştir. 4096 veri noktası 16 eş parçaya bölünmüştür. Bölünmüş bir EEG sinyali içerisinde toplam 256 adet veri kaydı bulundurmaktadır. Bu sayı her iki yöntem için aynidir.

EEG Sinyali 23.6 saniye

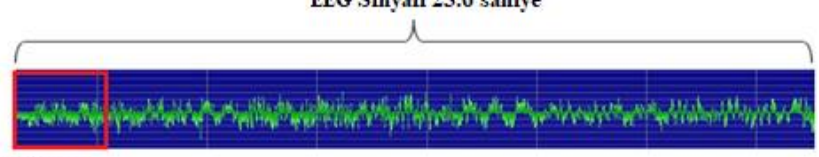

EEG Bölütleme

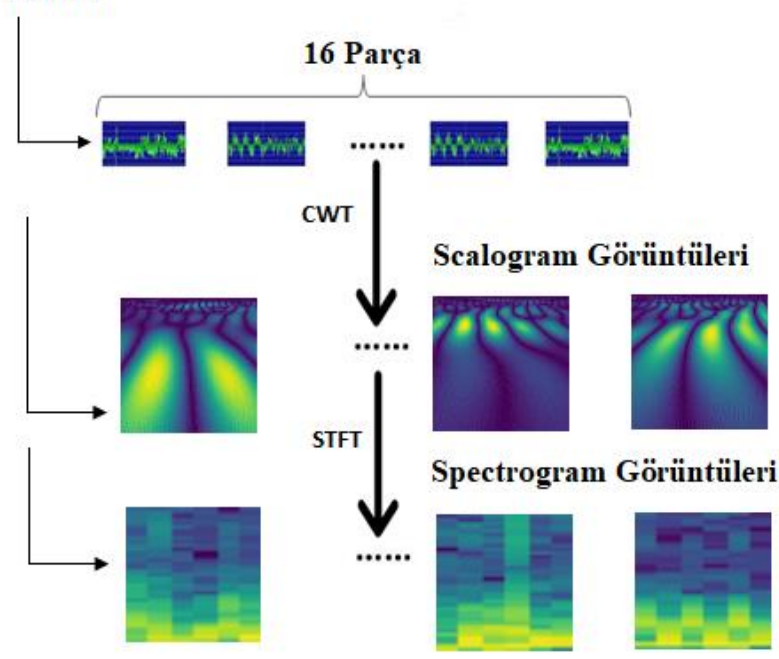

Şekil 3 EEG sinyal dönüşümleri. 


\subsection{Convolutional Neural Network (CNN)}

CNN tek, iki ya da üç boyutlu verilerde kullanılabilen bir derin öğrenme ağıdır. CNN genel olarak iki boyutlu verilerde özellikle görüntülerde kullanılmak için oluşturulmuştur (Indolia, Goswami, Mishra, \& Asopa, 2018). CNN yapıları içerisinde çeşitli katmanlar barındırmaktadır.

Convolution Katmanı: Bu katman CNN yapısı için oldukça önemlidir. Bu katman görüntüdeki özelliklerin tespit edilmesini sağlar. Görüntü verileri içerisinde düşük ya da yüksek dereceli özellikler barındırır. Bu özelliklerin algılanması için görüntüye filtre veya kernel adı verilen örnek bir matris uygulanır. Filtre matrisinin boyutları genellikle $3 \times 3,5 \times 5,7 \times 7$ gibi değerlerdedir. Filtre matrisi görüntünün sol üst köşesinden başlayarak görüntü boyunca kaydırılarak gezer. Filtre matrisi görüntü boyunca ilerlerken görüntü ve filtre matrisi değerleri indislerine göre birbirleri ile çarpılır ve elde edilen sonuçlar toplanır. Daha sonra toplam sonuç bir çıktı matrisine kaydedilir. Bu işlem tüm görüntü boyunca aynı şekilde devam ettirilir. Çıktı matrisine kaydedilen değerler sonucu oluşan matrise Feature Map denir. Eğer görüntü üç kanallı bir görüntü ise örnek olarak RGB bir görüntü ise tüm işlemler her bir kanal için yapılır ve sonuçlar bias değeri ile toplandıktan sonra çıktı matrisi elde edilir. $\mathrm{Bu}$ işleme Convolution denir. Denklem (3)'te Convolution işlemi gösterilmiştir. Giriş görüntüsü f ile ifade edilirken, filtre h ile ifade edilmiştir.

$$
\begin{gathered}
G[m, n]=(f * h)[m, n] \\
G[m, n]=\sum_{j} \sum_{k} h[j, k] f[m-j, n-k]
\end{gathered}
$$

Batch Normalization (BN) Katmanı: Derin öğrenme yapılarında her bir katman bir sonraki katmanın girdisi şeklinde çalışır. Bir katmandaki öğrenme işlemi bitmeden bir diğer katmanda öğrenme işlemi başlamaz. Normalizasyon işlemi ile girdi değerleri standartlaştırılır. Ancak başta yapılan normalizasyon işleminden ara katmanlar yararlanamaz. Bu yüzden eğitim daha yavaş, daha kararsız ya da gradyan kaybolması öğrenmenin minimum düzeyde gerçekleşmesi gibi problemlerde karşılaşılabilir (Alzubaidi et al., 2021). Tüm bu sorunların önüne geçmek adına BN kullanılır. BN uygulamanın model skorlarına olumlu yönde etkisi olduğu bilinmektedir (Alzubaidi et al., 2021).

Aktivasyon Katmanı: Bu katmana doğrusal olmayan katman da denilebilir. Bu katmanda doğrusal olmayan sigmoid, tanh, ReLU vb. aktivasyon fonksiyonları kullanılır. Aktivasyon fonksiyonları bir nöronun gelen girişe nasıl bir işlem uygulaması gerektiğini belirler ve böylece çıkışı oluşturur. Denklem (4)'te tanh, denklem (5)'te sigmoid ve denklem (6)'da ReLU aktivasyon fonksiyonun denklemi gösterilmiştir.

$$
\begin{gathered}
\operatorname{Tanh}(x)=\tanh (x)=\frac{\exp (x)-\exp (-x)}{\exp (+x)+\exp (-x)} \\
\operatorname{Sigmoid}(x)=\sigma(x)=\frac{1}{1+\exp (-x)} \\
\operatorname{ReLU}(x)=(x)^{+}=\max (0, x)
\end{gathered}
$$

Pooling Katmanı: Pooling Katmanı Convolution Katmanı ile benzer şekilde çalışır ve görüntü boyutlarını küçülterek verilerin hesaplanma yükünü azaltmaktadır (Indolia et al., 2018). Max Pooling ve Average Pooling olmak üzere iki çeşit pooling vardır. Max Pooling filtrenin/kernelin gezdiği kısmın maksimum değerini alır ve çıktı matrisinde depolar. Average Pooling ise filtrenin/kernelin kapsadığı kısmın ortalamasını alarak çıktı matrisinde depolar. Bu işlemi her iki pooling yöntemi de görüntü boyunca tekrarlayarak çıktı matrisini oluşturur. Max Pooling Katmanı ayrıca bir gürültü bastırıcı olarak çalışmaktadır. Gürültülü kısımlar tamamen ortadan kaldırılabilir. Bu yönü ile diğer pooling yönteminden daha iyi performans gösterebilir (Indolia et al., 2018).

Dropout Katmanı: $\mathrm{Bu}$ katman model eğitimi sırasında rastgele bir şekilde belirlenen oran miktarınca nöron birimlerinin in-aktif edilerek kullanılmamasını sağlamaktadır. Dropout Katmanı hem modelin aşırı öğrenmesini (over-fitting) önleyebilir hem de modelin iş yükünü azaltır (Gu et al., 2018).

Flatten Katmanı: Bu katman sayesinde veriler tek boyutlu bir dizi haline getirilir. Bundan sonra gelecek olan Fully Connected Katmanı'nın giriş verisi Flatten sayesinde hazırlanmış olur.

Fully Connected (FC) Katmanı: Flatten ile tek boyutlu dizi şekline getirilen veriler FC'nin giriş verisidir. FC'de her bir giriş tüm nöronlar ile bağlıdır.

\subsection{Recurrent Neural Network (RNN)}

RNN zaman serileri, doğal dil işleme ve sıralı veriler için kullanılmaktadır (Alzubaidi et al., 2021). Normalde klasik derin öğrenme ağlarında giriş ve çıkışlar birbirinden bağımsız bir şekildedir. Ancak RNN'lerde girdiler birbiriyle ilişkilidir. Bir önceki katmandaki girdilerden alınan çıktı hafızaya alınır. Yani RNN'lerin kısa vadeli bir hafizası vardır. Diğer katmanda girdiler ile bir girdi olarak kullanılır. RNN'de her çıkış bir önceki adıma bağımlıdır. Bir önceki girdiler sonraki katmanın çıktısını etkilemektedir. Ancak RNN'nin bu kısa vadeli hafızası bazı durumlarda yetersiz kalmaktadır. Ayrıca geriye yayılımda gradyan yok olması yani öğrenmenin minimum düzeyde kalmasina sebep olabilmektedir (Alzubaidi et al., 2021). Long Short-Term Memory (LSTM), RNN'nin bu eksikliklerinin önüne geçmek için ortaya çıkmıştır. RNN'de bir tane tanh katmanı varken LSTM'de Hafiza Hücresi, Unutma Kapısı, Giriş Kapısı ve Çıkış Kapısı olmak üzere dört farklı katman vardır (Chen, 2016). Hafıza Hücresi unutulmaması gereken önemli bilgiyi hücreler boyunca taşıyan iletim hattına ve ağ hafizasına verilen isimdir. Bu yöntem ile short-term memory probleminin önüne geçilir. Unutma Kapısı bir sigmoid fonksiyonu vasıtasıyla hangi bilgilerin hafızada tutulacağ 1 ve hangi bilgilerin unutulacağına karar verir. Hafiza Hücresi'nin, önceki ve sonraki bilginin sigmoid işleminin sonucu ve tanh işlemi sonucu çarpılarak güncellenip güncellenmeyeceğine Giriş Kapısı karar verir. Çıkış Kapısı, sonraki hücrenin girişini belirlemek için kullanılır. Çıkış Kapısı önceki bilgi ve mevcut girişin sigmoid fonksiyonu sonucu ile Hafiza Hücresinde var olan bilginin tanh fonksiyonundaki sonucu çarpılarak bir sonraki hücrenin giriş bilgilerine karar verir. Şekil 4'te LSTM'in içyapısındaki bileşenler gösterilmiştir.

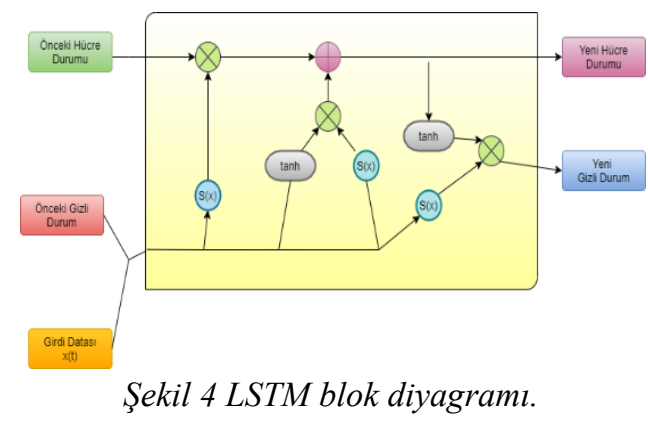

1213 


\subsection{D-CNN + RNN(LSTM) Kombine Modeli}

Bu çalışmada CNN ve RNN yapılarının ikisi de kullanılarak kombine bir sistem oluşturulmuştur. CNN bloğunda 5 katman CNN kullanılmıştır. RNN bloğu için LSTM yapısı kullanılmıştır. Daha sonra her iki blok düzleștirilerek birleștirilmiştir. Birleştirildikten sonra model sanki tek bir yapı gibi 3 tane FC katmanı eklenmiştir. Son olarak 2'li sınıflandırma işlemi Sigmoid aktivasyon fonksiyonu ile model çıktısı elde edilmiş̧ir. Model blok diyagramı Şekil 5'te gösterilmiştir. Modelde Dropout oranı olarak 0.2 kullanılmıştır. Optimizer olarak Adam kullanılmıştır. Batch_size olarak ise 4 seçilmiştir.

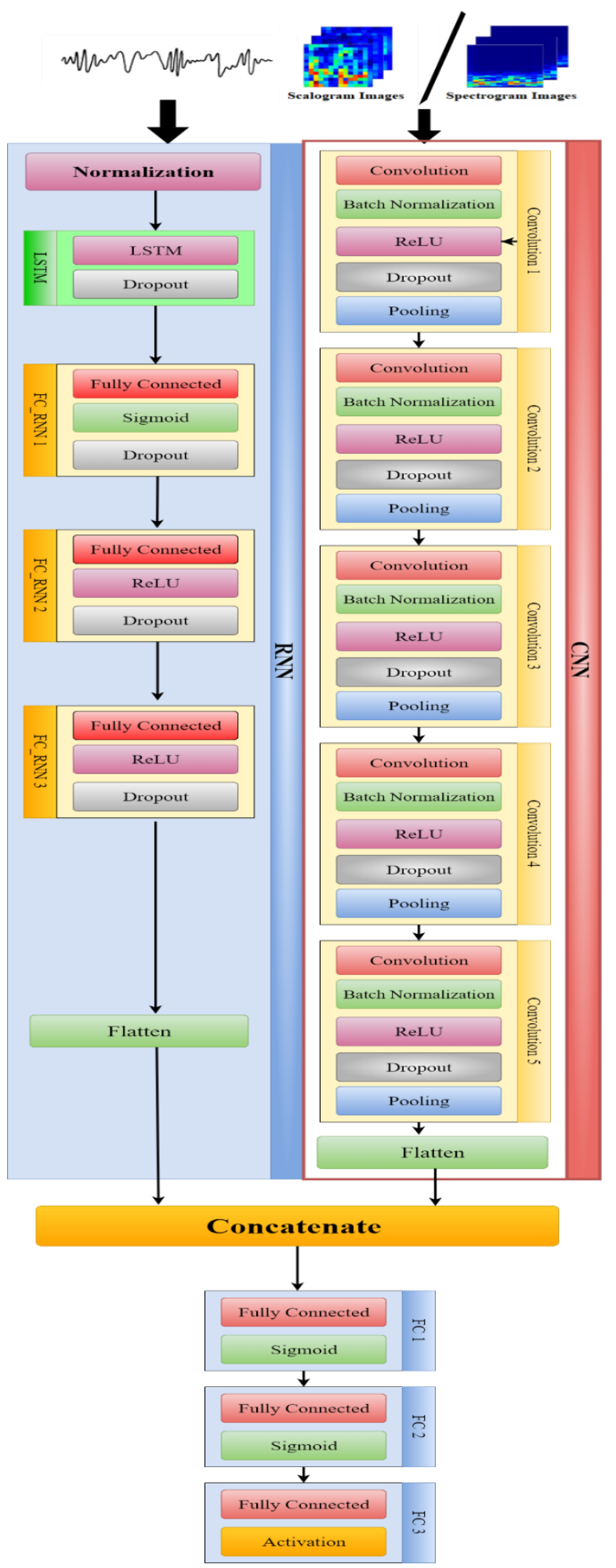

Şekil 5 Model blok diyagramı.

\section{Araştırma Sonuçları ve Tartışma}

$\mathrm{Bu}$ çalışmada EEG sinyalleri zaman-frekans görüntü dönüşümü ile sinyallerin frekans alanındaki görüntüleri ve EEG sinyallerinin kendisi kullanılarak kombine bir sistem oluşturulmuştur. Model, A, B, C, D setlerini epilepsi nöbeti geçirmediği durum, E setini ise epilepsi nöbet aktivitesi olarak tanımlayarak başarılı bir şekilde sınıflandırmıştır. Modelimizin başarısı çeşitli değerlendirme skorları ile değerlendirilmiş̧ir. Modelin CNN girdisi için spectrogram ve scalogram verileri ayrı ayrı denenmiş ve değerlendirilmiş̧ir. Modelimiz, CNN ve LSTM olmak üzere iki farklı derin öğrenme yapısını bir araya getirerek kabul gören sonuçlar alması açısından oldukça önemlidir. Tablo 2 'de modelimizin değerlendirme sonuçları verilmiştir.

Tablo 2 Model performans sonuçlart.

\begin{tabular}{c|c|c}
\hline Yöntem & Accuracy (\%) & F $_{\mathbf{1}}$ - Score (\%) \\
\hline $\begin{array}{c}\text { 2D CNN CWT + } \\
\text { LSTM }\end{array}$ & 99.27 & 99.27 \\
\hline $\begin{array}{c}\text { 2D CNN STFT + } \\
\text { LSTM }\end{array}$ & 99.47 & 99.48 \\
\hline
\end{tabular}

\begin{tabular}{c|c|c}
\hline Yöntem & Sensitivity (\%) & Specificity (\%) \\
\hline $\begin{array}{c}\text { 2D CNN CWT + } \\
\text { LSTM }\end{array}$ & 99.27 & 98.04 \\
\hline $\begin{array}{c}\text { 2D CNN STFT + } \\
\text { LSTM }\end{array}$ & 99.47 & 99.45 \\
\hline
\end{tabular}

\begin{tabular}{c|c|c}
\hline Yöntem & MCC (\%) & ROC AUC (\%) \\
\hline $\begin{array}{c}\text { 2D CNN CWT }+ \\
\text { LSTM }\end{array}$ & 97.73 & 99.94 \\
\hline $\begin{array}{c}\text { 2D CNN STFT + } \\
\text { LSTM }\end{array}$ & 98.37 & 99.97 \\
\hline
\end{tabular}

Sonuçlardan da anlaşılacağı üzere geliştirdiğimiz modellerden 2D CNN STFT + LSTM yöntemi yani CNN girdi verileri olarak spectrogram görüntülerini kullandığımız model $99.47 \%$ doğruluk oranına ulaşmıştır. Diğer yöntem olan 2D CNN CWT + LSTM modelimiz ise $99.27 \%$ doğruluk oranına ulaşmıştır. Diğer performans değerlendirme skorlarımızdan da anlaşıldığ sonuçlar almıştır. 2D CNN CWT + LSTM modeli 6400 epilepsi nöbet aktivitesi olmayan gözlemin 20 tanesini nöbet aktivitesi var olarak ve 1600 nöbet aktivitesi olan gözlemin 38 tanesini nöbet aktivitesi yok olarak sınıflandırmıştır. 2D CNN STFT + LSTM modeli 6400 epilepsi nöbet aktivitesi olmayan gözlemin 33 tanesini nöbet aktivitesi var olarak ve 1600 nöbet aktivitesi olan gözlemin 9 tanesini nöbet aktivitesi yok olarak sınıflandırmıştır.

Özellikle epilepsi olmak üzere birçok sinirsel hastalığın teşhisinde EEG sinyallerinden faydalanılmaktadır. Günümüzde makine öğrenmesi ve derin öğrenme yaklaşımlarının yaygınlaşması ve gelişmesinden kaynaklı sinyal işleme, sinyallerden özellik çıkarımı, sinyallerden otomatik hastalık tayini gibi birçok ilerleme meydana gelmiştir. Bu çalışmada epilepsi hastalı̆g üzerine otomatik nöbet tespiti üzerine 2D CNN CWT + LSTM ve 2D CNN STFT + LSTM modelleri geliştirilmiştir. Alınan sonuçlar neticesinde klinik açıdan önemli bir başarı elde edilmiştir. 2D CNN CWT + LSTM için sinyal zaman-frekans-görüntü dönüşümlerinden birisi olan CWT ile scalogram görüntüleri üzerine çalışılmıştır. 2D CNN STFT + 
LSTM için sinyal zaman-frekans-görüntü dönüşümlerinden birisi olan STFT ile spectrogram görüntüleri üzerine çalışılmıştır. Literatürde bu tarz sinyal görüntüsü ile çalışılmış örnekler mevcuttur. Çalışma kapsamında sadece sinyalin frekans alanı görüntüsü ile CNN yöntemi kullanılmayıp aynı zamanda sinyalin ham zaman dizisi hali bir RNN çeşidi olan LSTM yöntemi ile kullanılarak kombine bir model oluşturulmuştur. Modelimiz ikili sınıflandırma için oldukça tatmin edici sonuçlar elde etmiştir. Tablo 3'te daha önceden Bonn veri seti üzerine çalışılmış derin öğrenme ve makine öğrenmesi yaklaşımlarına ait performans değerlendirmelerinin önerdiğimiz model ile kıyaslaması mevcuttur.

Tablo 3 Bonn veri seti üzerine daha önceden yapılan dĭger çalışmalar ile önerilen yöntemin karşılaştırılması.

\begin{tabular}{|c|c|c|c|}
\hline Çalışma & Yll & Yöntem & $\begin{array}{c}\text { Accuracy } \\
(\%)\end{array}$ \\
\hline $\begin{array}{l}\text { (Sharmila \& } \\
\text { Geethanjali, } \\
\text { 2016) }\end{array}$ & 2016 & $\begin{array}{l}\text { DWT Based k-NN } \\
\text { Classifiers }\end{array}$ & 97.10 \\
\hline $\begin{array}{c}\text { (Sharmila \& } \\
\text { Geethanjali, } \\
\text { 2016) }\end{array}$ & 2016 & $\begin{array}{l}\text { DWT Based Naive } \\
\text { Bayes Classifiers }\end{array}$ & 95.85 \\
\hline $\begin{array}{l}\text { (MURSALIN, } \\
\text { ZHANG, } \\
\text { CHEN, \& } \\
\text { CHAWLA, } \\
\text { 2017) }\end{array}$ & 2017 & $\begin{array}{l}\text { Random Forest and } \\
\text { Correlation Based } \\
\text { Feature Selection }\end{array}$ & 97.40 \\
\hline $\begin{array}{c}\text { (Jaiswal \& } \\
\text { Banka, 2017) }\end{array}$ & 2017 & ANN and LNDP & 98.72 \\
\hline $\begin{array}{c}\text { (Jaiswal \& } \\
\text { Banka, 2017) }\end{array}$ & 2017 & 1D-LGP +ANN & 98.65 \\
\hline $\begin{array}{l}\text { (ZHAO ET } \\
\text { AL., 2020) }\end{array}$ & 2020 & 1D-CNN & 98.76 \\
\hline $\begin{array}{l}\text { (SHEORAN, } \\
\text { RATHEE, \& } \\
\text { SAİII, 2020) }\end{array}$ & 2020 & $\begin{array}{l}\text { BEMD and DML on } \\
\text { Scalogram }\end{array}$ & 99.08 \\
\hline \multirow[t]{3}{*}{$\begin{array}{l}\text { (WANG ET } \\
\text { AL., 2021) }\end{array}$} & 2021 & $\begin{array}{l}\text { Bi-LSTM-AM and 1D- } \\
\text { CNN }\end{array}$ & 92.07 \\
\hline & - & $\begin{array}{c}\text { Önerilen 2D CNN } \\
\text { STFT + LSTM }\end{array}$ & 99.47 \\
\hline & - & $\begin{array}{c}\text { Önerilen 2D CNN } \\
\text { CWT + LSTM }\end{array}$ & 99.27 \\
\hline Çalışma & Yll & Yöntem & $\begin{array}{c}\text { Sensitivity } \\
(\%)\end{array}$ \\
\hline $\begin{array}{l}\text { (Sharmila \& } \\
\text { Geethanjali, } \\
\text { 2016) }\end{array}$ & 2016 & $\begin{array}{l}\text { DWT Based k-NN } \\
\text { Classifiers }\end{array}$ & 93.62 \\
\hline $\begin{array}{c}\text { (Sharmila \& } \\
\text { Geethanjali, } \\
\text { 2016) }\end{array}$ & 2016 & $\begin{array}{l}\text { DWT Based Naive } \\
\text { Bayes Classifiers }\end{array}$ & 89.92 \\
\hline $\begin{array}{l}\text { (MURSALIN } \\
\text { ET AL., } \\
\text { 2017) }\end{array}$ & 2017 & $\begin{array}{l}\text { Random Forest and } \\
\text { Correlation Based } \\
\text { Feature Selection }\end{array}$ & 97.40 \\
\hline $\begin{array}{c}\text { (Jaiswal \& } \\
\text { Banka, 2017) }\end{array}$ & 2017 & ANN and LNDP & 98.30 \\
\hline \multirow[t]{3}{*}{$\begin{array}{c}\text { (Jaiswal \& } \\
\text { Banka, 2017) }\end{array}$} & 2017 & 1D-LGP +ANN & 98.44 \\
\hline & - & $\begin{array}{l}\text { Önerilen 2D CNN } \\
\text { STFT + LSTM }\end{array}$ & 99.47 \\
\hline & - & $\begin{array}{c}\text { Önerilen 2D CNN } \\
\text { CWT + LSTM }\end{array}$ & 99.27 \\
\hline
\end{tabular}

\begin{tabular}{|c|c|c|c|}
\hline Çalışma & Yll & Yöntem & $\begin{array}{c}\text { Specificity } \\
(\%)\end{array}$ \\
\hline $\begin{array}{l}\text { (Sharmila \& } \\
\text { Geethanjali, } \\
\text { 2016) }\end{array}$ & 2016 & $\begin{array}{l}\text { DWT Based k-NN } \\
\text { Classifiers }\end{array}$ & 97.94 \\
\hline $\begin{array}{l}\text { (Sharmila \& } \\
\text { Geethanjali, } \\
\text { 2016) }\end{array}$ & 2016 & $\begin{array}{l}\text { DWT Based Naive } \\
\text { Bayes Classifiers }\end{array}$ & 97.31 \\
\hline $\begin{array}{l}\text { (MURSALIN } \\
\text { ET AL., } \\
\text { 2017) }\end{array}$ & 2017 & $\begin{array}{l}\text { Random Forest and } \\
\text { Correlation Based } \\
\text { Feature Selection }\end{array}$ & 97.50 \\
\hline $\begin{array}{c}\text { (Jaiswal \& } \\
\text { Banka, 2017) }\end{array}$ & 2017 & ANN and LNDP & 98.82 \\
\hline \multirow[t]{3}{*}{$\begin{array}{c}\text { (Jaiswal \& } \\
\text { Banka, 2017) }\end{array}$} & 2017 & 1D-LGP +ANN & 98.70 \\
\hline & - & $\begin{array}{c}\text { Önerilen 2D CNN } \\
\text { STFT + LSTM }\end{array}$ & 99.45 \\
\hline & - & $\begin{array}{c}\text { Önerilen 2D CNN } \\
\text { CWT + LSTM }\end{array}$ & 98.04 \\
\hline
\end{tabular}

\section{Sonuç}

$\mathrm{Bu}$ çalışma da kullanılan yöntemler ile epilepsi nöbet aktivitesi yüksek doğruluk oranı ile tespit edilmiştir. Halka açık veri seti olan Bonn veri seti üzerinde kurulan model literatürde bulunan diğer çalışmalar ile rekabet edebilir sonuçlar almıştır. Çalışmamız, literatüre kazandırdığı yaklaşım ve aldığı yüksek skorlar açısından önemlidir. Çalışmamızda spectrogram görüntüleri ile alınan sonuçların scalogram görüntüleri ile alınan sonuçlardan daha iyi olduğu gözlemlenmiştir. Sonraki çalışmalarda modelin farklı veri setlerindeki performansının bulunması planlanmaktadır.

\section{Kaynakça}

Alzubaidi, L., Zhang, J., Humaidi, A. J., Al-Dujaili, A., Duan, Y., Al-Shamma, O., . . . Farhan, L. (2021). Review of deep learning: concepts, CNN architectures, challenges, applications, future directions. Journal of Big Data, 8(1), 53. doi:10.1186/s40537-021-00444-8

Andrzejak, R. G., Lehnertz, K., Mormann, F., Rieke, C., David, P., \& Elger, C. E. (2001). Indications of nonlinear deterministic and finite-dimensional structures in time series of brain electrical activity: dependence on recording region and brain state. Phys Rev E Stat Nonlin Soft Matter Phys, 64(6 Pt 1), 061907. doi:10.1103/PhysRevE.64.061907

Bajaj, N. (2020). Wavelets for EEG Analysis.

Beghi, E. (2020). The Epidemiology of Epilepsy. Neuroepidemiology, 54(2), 185-191. doi:10.1159/000503831

Brian, P., Avirath, S., Sean, C., Victoria, G., Antoni, V.-C., \& Adrien, M. (2021). Brain Informatics. doi:10.21203/rs.3.rs$112880 / \mathrm{v} 1$

Chen, G. (2016). A Gentle Tutorial of Recurrent Neural Network with Error Backpropagation.

Dwi Saputro, I. R., Maryati, N. D., Solihati, S. R., Wijayanto, I., Hadiyoso, S., \& Patmasari, R. (2019). Seizure Type Classification on EEG Signal using Support Vector Machine. Journal of Physics: Conference Series, 1201, 012065. doi:10.1088/1742-6596/1201/1/012065

Gu, J., Wang, Z., Kuen, J., Ma, L., Shahroudy, A., Shuai, B., . . . Chen, T. (2018). Recent advances in convolutional neural 
networks. Pattern Recognition, 77, 354-377. doi:https://doi.org/10.1016/j.patcog.2017.10.013

Hussain, L. (2018). Detecting epileptic seizure with different feature extracting strategies using robust machine learning classification techniques by applying advance parameter optimization approach. Cognitive neurodynamics, 12(3), 271294. doi:10.1007/s11571-018-9477-1

Indolia, S., Goswami, A. K., Mishra, S. P., \& Asopa, P. (2018). Conceptual Understanding of Convolutional Neural Network- A Deep Learning Approach. Procedia Computer Science, 132, 679-688. doi:https://doi.org/10.1016/j.procs.2018.05.069

Jaiswal, A. K., \& Banka, H. (2017). Local pattern transformation based feature extraction techniques for classification of epileptic EEG signals. Biomedical Signal Processing and Control, 34, 81-92. doi:https://doi.org/10.1016/j.bspc.2017.01.005

Kıymık, M. K., Güler, İ., Dizibüyük, A., \& Akın, M. (2005). Comparison of STFT and wavelet transform methods in determining epileptic seizure activity in EEG signals for realtime application. Computers in Biology and Medicine, 35(7), 603-616.

doi:https://doi.org/10.1016/j.compbiomed.2004.05.001

Mursalin, M., Zhang, Y., Chen, Y., \& Chawla, N. (2017). Automated epileptic seizure detection using improved correlation-based feature selection with random forest classifier. Neurocomputing, 241, 204-214.

Rajoub, B. (2020). Chapter 2 - Characterization of biomedical signals: Feature engineering and extraction. In W. Zgallai (Ed.), Biomedical Signal Processing and Artificial Intelligence in Healthcare (pp. 29-50): Academic Press.

Ravi Kumar, M., \& Srinivasa Rao, Y. (2019). Epileptic seizures classification in EEG signal based on semantic features and variational mode decomposition. Cluster Computing, 22(6), 13521-13531. doi:10.1007/s10586-018-1995-4

Sharmila, A., \& Geethanjali, P. (2016). DWT Based Detection of Epileptic Seizure From EEG Signals Using Naive Bayes and k-NN Classifiers. IEEE Access, 4, 7716-7727. doi:10.1109/ACCESS.2016.2585661

Sheoran, P., Rathee, N., \& Saini, J. S. (2020, 27-28 Feb. 2020). Epileptic Seizure Detection using Bidimensional Empirical Mode Decomposition and Distance Metric Learning on Scalogram. Paper presented at the 2020 7th International Conference on Signal Processing and Integrated Networks (SPIN).

Shi, X., Wang, T., Wang, L., Liu, H., \& Yan, N. (2019, 18-21 Nov. 2019). Hybrid Convolutional Recurrent Neural Networks Outperform CNN and RNN in Task-state EEG Detection for Parkinson's Disease. Paper presented at the 2019 AsiaPacific Signal and Information Processing Association Annual Summit and Conference (APSIPA ASC).

Shoka, A., Dessouky, M., El-Sherbeny, A., \& El-Sayed, A. (2019). Literature Review on EEG Preprocessing, Feature Extraction, and Classifications Techniques. Menoufia Journal of Electronic Engineering Research, 28(ICEEM2019-Special Issue), 292-299. doi:10.21608/mjeer.2019.64927

Siddiqui, M. K., Morales-Menendez, R., Huang, X., \& Hussain, N. (2020). A review of epileptic seizure detection using machine learning classifiers. Brain Informatics, 7(1), 5-5. doi:10.1186/s40708-020-00105-1

Singh, A., \& Trevick, S. (2016). The Epidemiology of Global Epilepsy. Neurologic clinics, 34(4), 837-847. doi:10.1016/j.ncl.2016.06.015 van Mierlo, P., Vorderwülbecke, B. J., Staljanssens, W., Seeck, M., \& Vulliémoz, S. (2020). Ictal EEG source localization in focal epilepsy: Review and future perspectives. Clinical Neurophysiology, 131(11), 2600-2616. doi:https://doi.org/10.1016/j.clinph.2020.08.001

Wang, Y., Dai, Y., Liu, Z., Guo, J., Cao, G., Ouyang, M., . . Z Zhao, G. (2021). Computer-Aided Intracranial EEG Signal Identification Method Based on a Multi-Branch Deep Learning Fusion Model and Clinical Validation. Brain sciences, 11(5). doi:10.3390/brainsci11050615

Xu, S., Wang, Z., Sun, J., Zhang, Z., Wu, Z., Yang, T., . . . Cheng, C. (2020). Using a deep recurrent neural network with EEG signal to detect Parkinson's disease. Annals of translational medicine, 8(14), 874-874. doi:10.21037/atm-20-5100

Zhao, W., Zhao, W., Wang, W., Jiang, X., Zhang, X., Peng, Y., . . .Zhang, G. (2020). A Novel Deep Neural Network for Robust Detection of Seizures Using EEG Signals. Computational and Mathematical Methods in Medicine, 2020, 9689821. doi:10.1155/2020/9689821 